\title{
Choosing of roughness parameters to describe the diffuse reflective and emissive properties of selected dielectrics
}

\author{
by L. Rozanski*, R. Majchrowski* and R. Staniek* \\ *Poznan University of Technology, 60-965 Poznań, ul. Piotrowo 2, Poland, leszek.rozanski@gmail.com, \\ radomir.majrowski@gmai.com, roman.staniek@put.poznan.pl
}

\begin{abstract}
The investigations has been performed in order to choose the specific roughness parameters which would inform the customer about the diffuse emissive and reflective characteristics of the adhesive tapes used in the thermographic measurements. To achieve that, a series of the surface topography parameters of various adhesive tapes (i.e. objects with diffusive reflective characteristics) and various glass plates (i.e. objects with directional reflective characteristics) has been examined. For the analysis of surface topography the following parameters were selected: Sdq (the Developed Interfacial Area Ratio) and Sdr (the Root Mean Square Surface Slope). These selected parameters best describe the properties of the surface in the discussed aspect.
\end{abstract}

\section{Introduction}

Remote temperature sensing and thermal imaging systems are invaluable tools in various fields of science and technology. The fact that radiation is a function of object surface temperature makes it possible for a remote temperature measurement systems to calculate this temperature. However, the radiation measured by these measuring systems does not only depend on the object temperature but is also a function of its emissivity. Therefore, to measure the temperature accurately with IR system, it is necessary to know this parameter. Emissivity is one of the major sources of error in radiometric measurements. In the radiometric temperature measurements which require determination of the emission coefficient of the object, in industrial conditions are widely applied special adhesive tapes. Those measuring methods are described in the literature. It is agreed that the emission coefficient of those tapes should be larger or equal to 0.95 , and this parameter is typically specified by the tape producer. Moreover, they have to have characteristics of the diffusion reflectors/emitters, but no manufacturer provide this kind of information. Thus, the investigations has been performed in order to choose the specific roughness parameters which would inform the customer about the diffuse reflective and emissive characteristics of the adhesive tapes used in the thermographic measurements.

\section{Emissive properties of materials}

Emissivity describes the object's ability to emit thermal radiation. Normally, object materials and surface treatments exhibit emissivity's ranging from approximately 0.05 to 0.99 . A highly polished surface falls below 0.1 , while an oxidised or painted surface has much higher emissivity.

Generally, emissivity is not constant, as it depends on several parameters: temperature, viewing angle, wavelength, contamination or roughness [1,2,3]. All total radiative properties of materials can only be regarded as function of viewing angle and temperature. Spectral emissivity as a function of wavelength decreases for metals, increases for dielectrics and is band - like for gases, liquids and same solids. A general characteristic, independent of the kind of material, is the variability of emissivity according to surface roughness. Emissivity increases with the increase of roughness. In particular the emissivity of metal, which is usually low, can considerably increase with roughness. Classification of emissivity models has been created under Fresnels's equation, Kirchhoff's low, Drude free electron theory (optically smooth surfaces) as well as applying bidirectional reflectance function (BRDF), geometric optics, and electromagnetic scattering theory (rough surfaces).

\section{Research method - surface topography analysis}

In the world around us, all the surfaces are rough. Most of surfaces are very complicated, and in order to describe it with certain values, the measurement and analysis of some parameters should be performed. Before 1980 roughness analysis was based on 2D measurements, which gave two-dimensional characteristics of the surface. In last decades, the metrology of the surface layer notes dynamical development as a science. This trend is the result of new technologies, especially in the motor, aircraft or electronic industry. During the last decades, many scientists and constructors became convinced that the third dimension should be added to the analysis. At present, 3D analysis of the surface geometry is widely accepted. In 2002, the ISO technical committee TC213 voted the creation of a new working group and assigned it the task of developing future international standards for areal surface texture. They developed a in which and the 3D parameters are defined (ISO 25178, 2008: Geometrical product specifications - Surface texture: Areal Part 2: Terms, definitions and surface texture parameters). 
The 3D parameters are divided into groups:

- $\quad$ amplitude parameters: Sa, Sq, Ssk, Sku, Sp, Sv, Sz,

- functional parameters: Smr, Smc,

- $\quad$ spatial parameters: Sal, Str, Std,

- $\quad$ hybrid parameters: Sdq, Sdr,

- $\quad$ functional parameters: $V m, V v, V m p, V m c, V v c, V v v$,

- $\quad$ feature parameters: Spd, Spc, S10z, S5p, S5v, Sda, Sdv, Sha, Shv.

For surface topography analysis are defined a set of functional indices making it possible to characterize surface zones involved in wear or contact phenomena. The useful tool is the polar spectrum. The Fourier spectrum, when it is integrated in polar coordinates makes it possible to determine the privileged direction of surface structures. The polar spectrum takes into account the power spectrum of the surface in each direction. The angle with the largest power spectrum corresponds to the privileged texture direction. The representation of the polar spectrum clearly shows the privileged directions. The polar spectrum is use to determine and quantified the value of surface isotropy. A surface is said to be isotropic when it presents identical characteristics regardless of the direction of measurement.

\section{Results and discussion}

There are many methods for available for geometric and surface topography measurements: contact and noncontact, micro and nanoscale approaches [4]. We have tested the application of white light interferometry for this purpose - optical surface profiler Veeco NT1100. The Vertical Scanning Interferometry (VSI) principle is realized by continuous vertical movement of the objective, the area of maximal interference corresponds whit subsequent examined levels. During operation, the system creates an interferogram from the examined surface, and the image is transmitted into CCD matrix [5]. A topographic map of the examined surface is produced, so that is one scanning path, depending on the type of objective, a surfaces up to $1 \times 1 \mathrm{~mm}$ may be analyzed. Larger areas may also be scanned, but the stitching of smaller scans is required. Four samples were tested Four samples were tested using a device NT100: two glass surfaces and two tape surfaces. For all surfaces the measurement areas were $0.9 \times 1.2 \mathrm{~mm}, 0,2 \times 0,3 \mathrm{~mm}$ and $0,09 \times 0,12$ $\mathrm{mm}$. For each area, five measurements of surface topography were made(a total of $4 \times 3 \times 5=60$ measurements). Sample images of the measured surface topography shown in Figures 1 and 2. Examples of surface topography analysis are shown in the Figures 3 and 4.
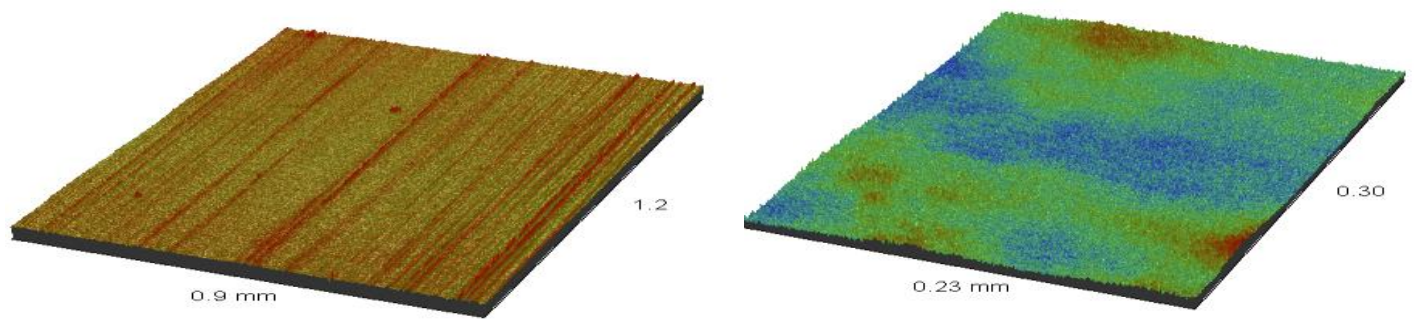

Fig. 1. Sample images of surface topography of glass, measurement areas: $0.9 \times 1.2 \mathrm{~mm}$ and $0,2 \times 0,3 \mathrm{~mm}$
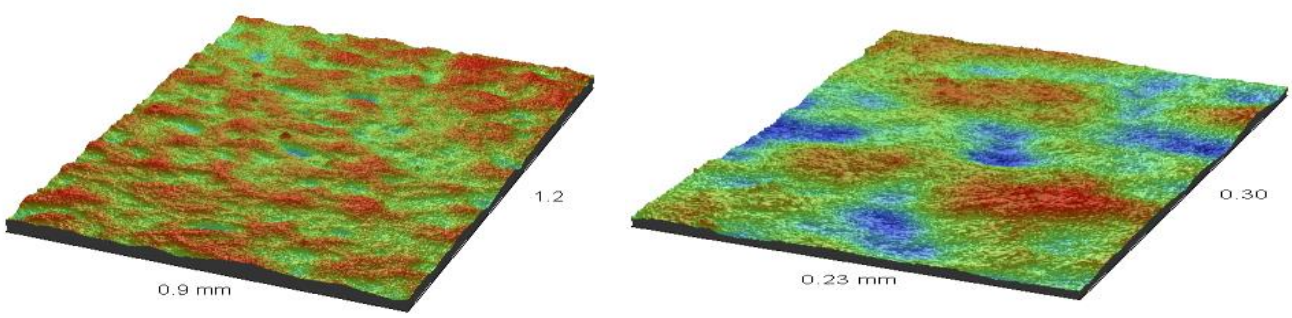

Fig. 2. Sample images of surface topography of tape, measurement areas: $0.9 \times 1.2 \mathrm{~mm}$ and $0,2 \times 0,3 \mathrm{~mm}$ 

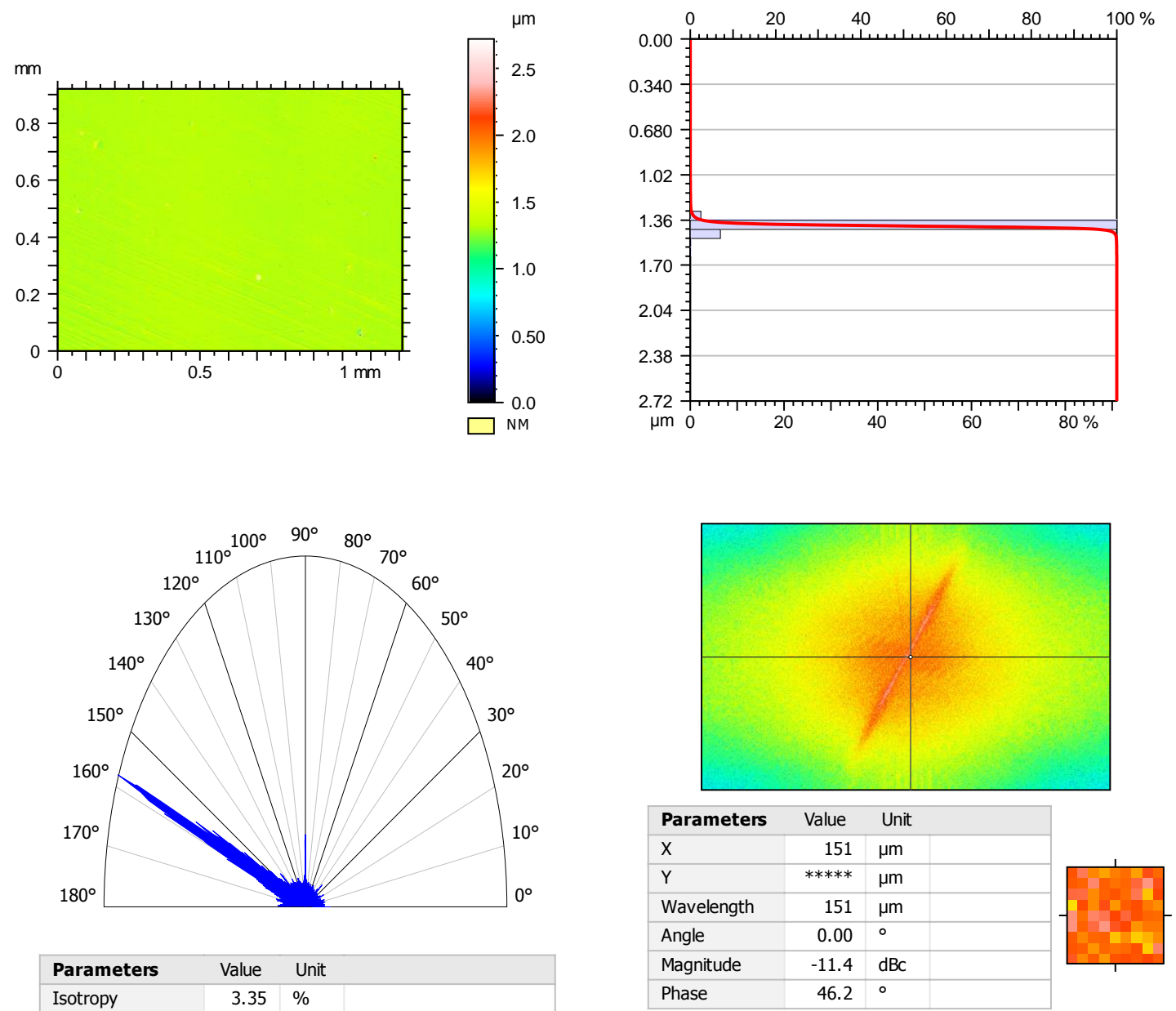

\begin{tabular}{|l|r|l|l|}
\hline Parameters & Value & \multicolumn{2}{l|}{ Unit } \\
\hline Isotropy & 3.35 & $\%$ & \\
\hline First Direction & 158 & $\circ$ & \\
\hline Second Direction & 153 & ${ }^{\circ}$ & \\
\hline Third Direction & 164 & $\circ$ & \\
\hline
\end{tabular}

\begin{tabular}{|l|l|l|l|l|l|l|l|}
\hline \multicolumn{2}{|l|}{ Amplitude parameters } & \multicolumn{2}{l|}{ Functional parameters } & \multicolumn{2}{l|}{ Spatial parameters } & \multicolumn{2}{l|}{ Hybrid parameters } \\
\hline Sq & $0.01 \mathrm{um}$ & Sbi & 0.57 & Sdr & $0.00 \%$ & Std & $90 \mathrm{deg}$ \\
\hline Sz & $0.17 \mathrm{um}$ & Svi & 0.08 & Sdq & $415 \mathrm{mdeg}$ & Sal & $0.01 \mathrm{~mm}$ \\
\hline Sku & 8.69 & Sci & 1.70 & Sds & $4.39 / \mathrm{mm}$ & Str & 0.02 \\
\hline Ssk & 1.07 & - & - & - & - & - & - \\
\hline
\end{tabular}

Fig. 3. Sample images of surface topography analysis of glass 



\begin{tabular}{|l|r|l|l|}
\hline Parameters & Value & Unit \\
\hline Isotropy & 87.7 & $\%$ & \\
\hline First Direction & 90.0 & $\circ$ & \\
\hline Second Direction & 0.144 & $\circ$ & \\
\hline Third Direction & 37.2 & $\circ$ & \\
\hline
\end{tabular}

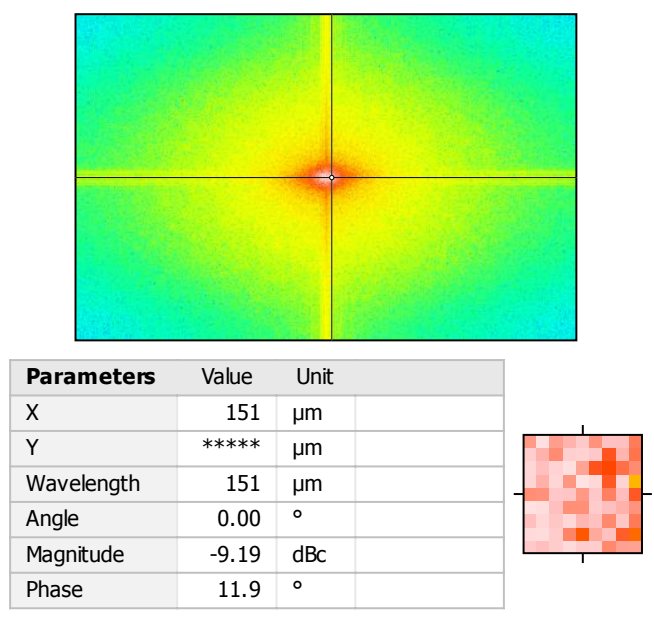

\begin{tabular}{|l|l|l|l|l|l|l|l|}
\hline \multicolumn{2}{|c|}{ Amplitude parameters } & \multicolumn{2}{l|}{ Functional parameters } & \multicolumn{2}{l|}{ Spatial parameters } & \multicolumn{2}{l|}{ Hybrid parameters } \\
\hline Sq & $1.38 \mathrm{um}$ & Sbi & 0.66 & Sdr & $1.46 \%$ & Std & - \\
\hline Sz & $9.80 \mathrm{um}$ & Svi & 0.13 & Sdq & $9.78 \mathrm{deg}$ & Sal & $53.74 \mathrm{~mm}$ \\
\hline Sku & 4.09 & Sci & 1.39 & Sds & $129 / \mathrm{mm}$ & Str & 0.82 \\
\hline Ssk & -0.46 & - & - & - & - & - & - \\
\hline
\end{tabular}

Fig. 4. Sample images of surface topography analysis of glass 
For the analysis of surface topography the following parameters were selected: Sdq, Sdr. These selected parameters best describe the properties of the surface in the discussed aspect. They are defined as [6]:

Sdr - the Developed Interfacial Area Ratio (1.1), is expressed as the percentage of additional surface area contributed by the texture as compared to an ideal plane the size of the measurement region. In the case of a flat surface is 0 , the rough $\mathrm{Sdr}>0$ and depends on the complexity of the surface;

$$
S d r=\frac{\sum_{j=1}^{N-1} \sum_{i=1}^{M-1} A_{i j}-(M-1)(N-1) \Delta x \Delta y}{(M-1)(N-1) \Delta x \Delta y} \cdot 100 \%
$$

where:

$$
\begin{aligned}
A_{i j}= & \frac{1}{4}\left\{\left(\left[\Delta y^{2}+\left(\eta\left(x_{i}, y_{j}\right)-\eta\left(x_{i}, y_{j+1}\right)\right)^{2}\right]^{1 / 2}+\left[\Delta y^{2}+\left(\eta\left(x_{i+1}, y_{j+1}\right)-\eta\left(x_{i+1}, y_{j+1}\right)\right)^{2}\right]^{1 / 2}\right) .\right. \\
& \left.\left(\left[\Delta x^{2}+\left(\eta\left(x_{i}, y_{j}\right)-\eta\left(x_{i+1}, y_{j}\right)\right)^{2}\right]^{1 / 2}+\left[\Delta x^{2}+\left(\eta\left(x_{i}, y_{j+1}\right)-\eta\left(x_{i+1}, y_{j+1}\right)\right)^{2}\right]^{1 / 2}\right)\right\}
\end{aligned}
$$

Sdq - the Root Mean Square (1.2) Surface Slope comprising the surface, evaluated over all directions (in the discussed aspect: small angles to better reflect light).

$$
S_{\Delta} q=\sqrt{\frac{1}{(M-1)(N-1)} \sum_{j=2}^{N} \sum_{i=2}^{M}\left[\left(\frac{\eta\left(x_{i}, y_{j}\right)-\eta\left(x_{i-1}, y_{j}\right)}{\Delta x}\right)^{2}+\left(\frac{\eta\left(x_{i}, y_{j}\right)-\eta\left(x_{i}, y_{j-1}\right)}{\Delta y}\right)^{2}\right]}
$$

The values of Sdr, Sdq and isotropy for the measured surface are shown in Table 1, Figure 5 is showing the average values of these parameters for the analyzed surface.

Table 1. The values of Sdr, Sdq and isotropy

\begin{tabular}{|c|c|c|c|c|c|c|}
\hline & \multicolumn{3}{|c|}{ GLASS I } & \multicolumn{3}{c|}{ GLASS II } \\
\hline No. measured area & Sdr [\%] & Sdq [deg] & Isotropy & Sdr [\%] & Sdq [deg] & Isotropy \\
\hline 1 & 0.003 & 0.433 & 0.820 & 0.001 & 0.225 & 21.700 \\
\hline 2 & 0.005 & 0.600 & 2.840 & 0.001 & 0.222 & 22.900 \\
\hline 3 & 0.008 & 0.739 & 3.350 & 0.001 & 0.279 & 25.450 \\
\hline 4 & 0.004 & 0.492 & 2.600 & 0.001 & 0.218 & 25.300 \\
\hline 5 & 0.004 & 0.508 & 8.400 & 0.001 & 0.218 & 25.200 \\
\hline & & & & & & \\
\hline arithmetic average & 0.005 & 0.554 & 3.602 & 0.001 & 0.232 & 24.110 \\
\hline standard deviation & 0.002 & 0.119 & 2.846 & 0.000 & 0.026 & 1.708 \\
\hline range & 0.005 & 0.306 & 7.580 & 0.000 & 0.062 & 3.750 \\
\hline & \multicolumn{7}{|c|}{ TAPE I } & & TAPE II \\
\hline No. measured area & Sdr [\%] & Sdq [deg] & Isotropy & Sdr [\%] & Sdq [deg] & Isotropy \\
\hline 1 & 1.458 & 9.774 & 58.200 & 2.049 & 11.578 & 56.200 \\
\hline 2 & 1.518 & 9.971 & 37.400 & 2.795 & 13.609 & 59.200 \\
\hline 3 & 1.554 & 10.090 & 44.000 & 2.451 & 12.730 & 75.700 \\
\hline 4 & 1.484 & 9.866 & 39.800 & 2.489 & 12.773 & 68.000 \\
\hline 5 & 1.460 & 9.784 & 87.700 & 2.513 & 12.859 & 55.600 \\
\hline & & & & & & \\
\hline arithmetic average & 1.495 & 9.897 & 53.420 & 2.459 & 12.710 & 62.940 \\
\hline standard deviation & 0.041 & 0.134 & 20.790 & 0.267 & 0.727 & 8.685 \\
\hline range & 0.096 & 0.316 & 50.300 & 0.747 & 2.030 & 20.100 \\
\hline
\end{tabular}




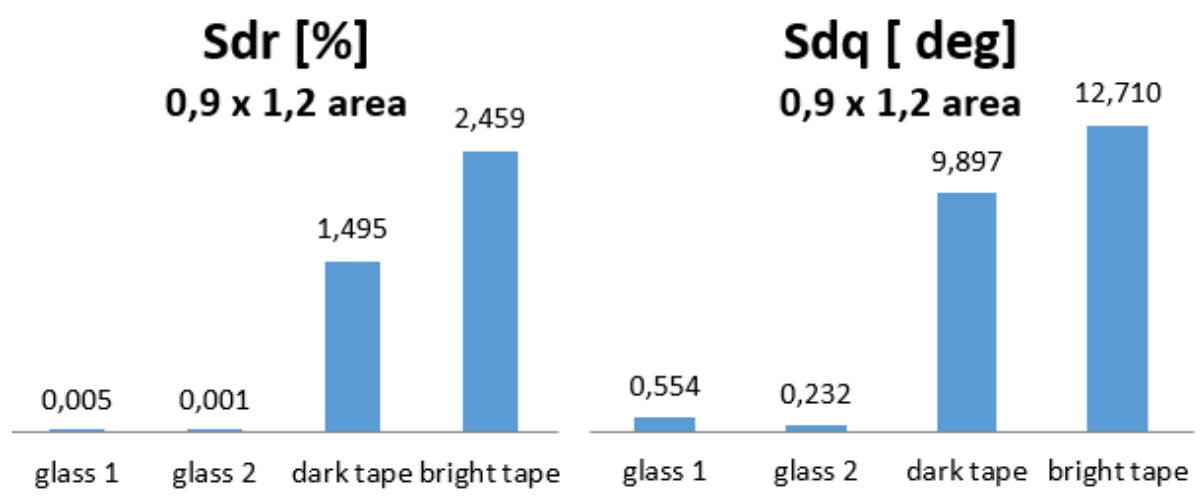

Fig. 5. Average values of selected parameters for the analyzed surface

\section{Conclusion}

The performed investigations proved with no doubts that the useful parameter corresponding with those characteristics could be a parameters Srd or Sdq. In case of the glass plates, their values are almost zero (very smooth surface), and in case of the adhesive tapes their values are larger than 1 (surface with many micro cavities and valleys).

\section{REFERENCES}

[1] Maldague X., "Theory and Practice of Infrared Technology for Nondestructive Testing". John Willey \& Sons, Inc., ISBN 0-471-18190-0, 2001.

[2] Chrzanowski K., "Non-contact thermometry-Measurement errors". SPIE PL, Research and Development Treaties, Warsaw, 2000.

[3] Chrzanowski K., "Problem of Determination of Effective Emissivity of some Materials in MIR Region". Infrared Phys. Technol., vol 30, pp. 679-684, 1995.

[4] Mathia T., Zahouani H., Stout K. J., Sullivan P.J., Dong W.P., Mainsah E., Lou N., "The development of methods for characterization of roughness in three dimensions". ECSC-EEC-EAEC, Brussels-Luxembourg and Authors, 1993.

[5] Schmit J. ,"High speed measurements using optical profiler". Veeco Instruments Inc., 2650 E. Elvira Road, Tucson, AZ, USA.

[6] Thomas T.R., "Rough Surfaces", Imperial College Press., Second Edition,1999. 